\title{
Gestión y vigilancia sanitaria de la fauna silvestre en el Perú
}

\author{
Wildlife health management and surveillance in Peru \\ Roberto Elías ${ }^{1,2}$, Raúl Berenguel ${ }^{1,2}$, Yuri Beraún ${ }^{3}$ Claudia Enrique ${ }^{3}$, Pedro Vásquez ${ }^{4}$
}

\section{RESUMEN}

Las enfermedades infecciosas son una seria amenaza para la conservación de la vida silvestre. En los últimos años, el crecimiento poblacional y los cambios ambientales han incrementado notablemente el acercamiento y el contacto de las personas con la fauna silvestre, lo cual junto al cambio de uso de las tierras han alterado el balance ecológico entre patógenos y sus hospederos humanos y animales, por eso la gestión y vigilancia sanitaria de la fauna silvestre deben implementarse en un sentido más amplio e integral que el que se realiza actualmente en nuestro país, por lo que urge la necesidad de promover las reformas necesarias para reorganizar y fortalecer la institucionalidad vinculada a estas.

PALABRAS CLAVE: Fauna silvestre, gestión sanitaria, vigilancia sanitaria, enfermedades.

\section{SUMMARY}

Infectious diseases are a serious threat to the conservation of wildlife. In recent years, population growth and environmental changes have significantly increased the frequency of contact of people with wildlife, which together with the change in land use have altered the ecological balance between pathogens and their human hosts. For this reason, the health management and monitoring of wildlife must be implemented in a broader and more comprehensive sense than that currently carried out in our country, which is why there is an urgent need to promote the necessary reforms to reorganize and strengthen of the institution linked to these.

KEY WORDS: Wildlife, health management, health surveillance, diseases.

\section{INTRODUCCIÓN}

Las principales amenazas a la fauna silvestre son la destrucción de los hábitats, la introducción de especies exóticas invasoras, la contaminación ambiental, el cambio climático, la sobreexplotación y comercio ilegal de especies, y la aparición de enfermedades (International Union for Conservation of Nature(UICN), 2009) siendo muchos de estos factores causados por acciones humanas.

Entre estos factores, las enfermedades infecciosas son una seria amenaza para la conservación de la vida silvestre. En los últimos años, el gran crecimiento poblacional y los cambios ambientales han incrementado notablemente el acercamiento y el contacto de las personas con la fauna silvestre, lo cual junto al cambio de uso de las tierras han alterado el balance ecológico entre patógenos y sus hospederos humanos y animales (Cunningham, Daszak \& Wood, 2017; Uhart et al., 2013). Estas interacciones han facilitado la infección de múltiples especies por un mismo patógeno, pudiendo afectar a personas, animales domésticos y silvestres (Cunningham et al., 2017).

\footnotetext{
Facultad de Medicina Veterinaria y Zootecnia, Universidad Peruana Cayetano Heredia. Lima, Perú.

Facultad de Ciencias de la Salud, Universidad Peruana de Ciencias Aplicadas . Lima, Perú.

Dirección General de Diversidad Biológica, Ministerio del Ambiente. Lima, Perú.

Facultad de Ciencias Forestales, Universidad Nacional Agraria La Molina. Lima, Perú.
} 
En las últimas décadas, se ha visto un incremento en la aparición de enfermedades infecciosas (Fisher et al., 2012). Muchas de estas, como la quitridiomicosis, el síndrome de la nariz blanca, o la enfermedad micótica de las serpientes han afectado de manera específica y muy grave distintas especies de fauna silvestre (Skerrat et al., 2007; Alves, Terribile \& Brito, 2014; Lorch et al., 2016).

De igual modo, existen enfermedades que han sido transmitidas de animales domésticos a animales de vida libre y que han puesto en peligro la conservación de especies silvestres. Un ejemplo de esto fue el brote de distemper canino que provocó la muerte de leones africanos (Panthera leo) en el Parque Nacional Serengueti en 1994 (Viana et al., 2015).

Otras enfermedades también compartidas por especies domésticas y silvestres, como la fiebre aftosa, la enfermedad de Newcastle, la fiebre porcina africana o la tuberculosis bovina pueden tener un impacto socioeconómico importante y desde el enfoque de la conservación de especies (Organización Mundial de Sanidad Animal (OIE), 2019; OIE, 2018; OIE, 2013; Weaver, Domenech, Thiermann \& Karesh, 2013).

Por otro lado, la mayoría de las enfermedades infecciosas son de origen zoonótico y más del $60 \%$ de ellas provienen de animales silvestres (Jones et al., 2008). Muchas de estas tienen mucha relevancia en la salud pública y significan una importante carga económica a nivel mundial (OIE, 2009). De igual manera, Cunningham et al. (2017) mencionan que las acciones antropogénicas como la destrucción de los hábitats y la diseminación de patógenos son una amenaza para la salud humana y de la biodiversidad.

En la actualidad, enfermedades como la rabia, el síndrome respiratorio agudo severo (SARS), o el síndrome respiratorio de Oriente Medio (MERS) son zoonosis muy importantes en salud pública (Ye et al., 2020) en las que están involucradas distintas especies de fauna silvestre y animales domésticos.

Asimismo, otras enfermedades como la enfermedad de caquexia crónica (WCD) que afecta a cérvidos o la enfermedad priónica de los camélidos son potenciales zoonosis que deberían ser consideradas dentro de los sistemas de vigilancia epidemiológica (Babelhadj et al., 2018; Barria et al., 2018, Libori, Mitchell \& Head., 2018; Race et al., 2014).
La situación pandémica actual, con la aparición del SARS CoV-2, ha involucrado inclusive a animales de compañía y silvestres. Existen estudios que sugieren que gatos domésticos, murciélagos y pangolines han podido participar en la transmisión de esta enfermedad (Lam et al., 2020; Shi et al., 2020; Zhang, Wu \& Zhang et al., 2020). Estos hechos, muestran la necesidad de implementar planes de vigilancia epidemiológica que aseguren y promuevan la salud pública y la conservación de los ecosistemas y de las especies de fauna silvestre con el fin de mitigar la aparición de estas amenazas, desde el enfoque holístico de Una Salud (Cunningham et al., 2017).

Finalmente, esta coyuntura pone en evidencia algunas carencias como errores en la notificación obligatoria de enfermedades, por parte de las autoridades locales, y la falta de información y la mala comunicación de este tipo de eventos; lo que puede causar pánico en las personas, estigmatizando y poniendo en peligro a especies de fauna silvestres, como sucedió en la ciudad de Cajamarca, en donde se atacó con fuego a murciélagos por el temor de contagiarse con SARS-CoV-2 (SPDA, 2019).

\section{ANTECEDENTES}

El Perú, considerado como uno de los 20 países megadiversos, no está libre de las enfermedades en la fauna silvestre. En nuestro país, se ha reportado la presencia de agentes patógenos causantes de enfermedades de notificación obligatoria ante la Organización Mundial de Sanidad Animal (OIE), las cuales tienen importancia desde el punto de vista de la salud animal y la salud pública; tal es el caso de la rabia, la influenza aviar, el hantavirus y la quitridiomicosis, por mencionar algunas.

La revisión sobre la situación actual de la rabia en el país efectuada por Navarro, Bustamante \& Sato (2007), refiere que desde los estudios de Aurelio Málaga Alva en 1954, se capturaban vampiros comunes (Desmodus rotundus) en las islas guaneras, acantilados de la costa desértica, valles templados de la sierra, vertientes orientales de los Andes, bosques tropicales y en toda la selva amazónica; con el fin de evidenciar que la rabia era transmitida por los murciélagos. Esta asunción fue convalidada en 1969, con la confirmación del primer brote de rabia bovina en el departamento de Junín. En la actualidad, la rabia es considerada una enfermedad endémica del Perú, manifestada en dos ciclos: uno urbano relacionado a la transmisión por canes y otro silvestre causado principalmente por la mordedura 
de murciélagos hematófagos (Navarro et al., 2007). Debido a su condición de zoonosis, el Ministerio de Salud (MINSA) ha regulado el control de esta enfermedad bajo la Norma Técnica para la Prevención y Control de Rabia Humana (2006), que especifica las acciones que se deben realizar ante la sospecha de brotes en animales silvestres, especialmente murciélagos.

Por otro lado, en el Perú se han reportado otras enfermedades zoonóticas como la hantavirosis, que se transmite a los humanos principalmente por la inhalación de aerosoles generados por la orina y las heces de roedores infectados. En la ciudad de Iquitos, Powers y colaboradores (1996) identificaron una variante del hantavirus en roedores silvestres (Oligoryzomys microtis), que fue reportada por primera vez en Bolivia (1996) bajo la denominación de Hantavirus río Mamoré (RIOMV); mientras que en julio del 2011, se informó que Iquitos presentaba los dos primeros casos de hantavirosis humana para el Perú, causados por el Hantavirus Seoul (SEOV) (Casapía et al., 2012).

En el 2009, Ghersi y colaboradores reportaron cuatro cepas de baja patogenicidad del virus de la Influenza Aviar (IA) aisladas de heces de aves marinas y costeras entre el 2006 y 2007 en el departamento de Lima (subtipos H3N8, H4N5, H10N9 y H13N2). Asimismo, en el 2013 Segovia y colaboradores lograron aislar siete cepas de baja patogenicidad de este virus (seis cepas procedentes de la especie migratoria Arenaria interpres y una de la especie residente Fulica ardesiaca). Como se sabe, el reservorio natural de los virus de la IA son las aves acuáticas silvestres, las cuales pueden estar infectadas con cepas del virus "avirulentas" o "poco patógenas" y presentar sólo signos clínicos leves o ningún signo. Los virus de baja patogenicidad pueden infectar aves domésticas y otros huéspedes intermedios, y convertirse en virus altamente patógenos (generalmente el subtipo H5N1), ocasionando enfermedades altamente letales (Rezza, 2004).

En el caso de los anfibios, en el 2016 se reportó la presencia del hongo Batrachochytrium dendrobatidis (Bd) en ranas altoandinas del género Telmatobius en nuestro país. Este agente, que ha infectado más de 500 especies de anfibios y ha disminuido las poblaciones de muchos de ellas en el mundo (Olson et al., 2013; Berger et al., 2016), fue reportada por Zevallos, Elías, Berenguel, Weaver \& Reading R. (2016) en animales decomisados en la ciudad de Lima, así como en ejemplares de T. culeus en vida libre en el lado peruano del lago (Berenguel et al., 2016). El género de ranas Telmatobius es uno de los más amenazados

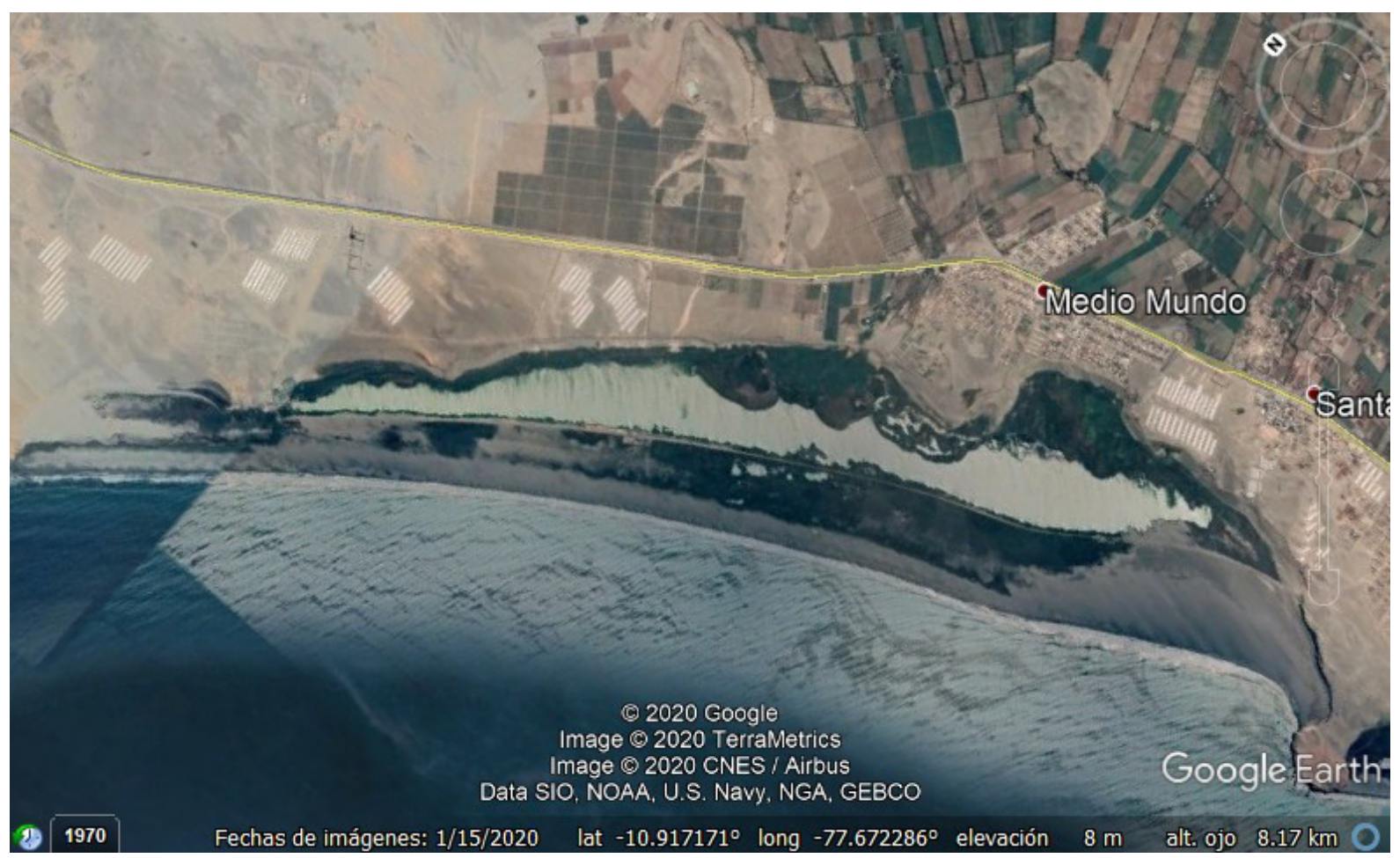

Figura 1. Laguna Medio Mundo, uno de los humedales más importantes de la costa central peruana y lugar de descanso de aves migratorias, con casi un centenar de galpones en las cercanías 
de Sudamérica: el $85 \%$ de las 63 especies están en alguna categoría de amenaza y el $\mathrm{Bd}$ es un riesgo potencial para su supervivencia.

Como se puede notar, la importancia de las enfermedades de la fauna silvestre no solo radica en el impacto que pueden generar sobre la salud animal, sino también sobre la salud humana; por ello, la relevancia de gestionar y vigilar sanitariamente a este grupo de especies.

En los últimos años, nuestro país ha sido testigo el origen de eventos de mortandad importantes vinculados con animales silvestres, los cuales pudieron estar asociados a factores sanitarios, aunque concluyeron sin ningún diagnóstico definitivo. Entre febrero y abril del 2012, el Instituto del Mar del Perú (IMARPE) reportó la mortandad de delfines (principalmente Delphinus capensis) entre las penínsulas de Illescas (Piura) y Chérrepe (La Libertad), llegando a contabilizar hasta 877 especímenes varados; sin embargo, a pesar de sus esfuerzos, no se llegó a determinar la causa específica de muerte de estos animales («Mortandad de delfines en el litoral de la costa norte, febrero a abril de 2012. Informe final.», 2012). Asimismo, en el 2016, 10 mil ranas del Titicaca (Telmatobius culeus) fueron reportadas muertas por la Administración Técnica Forestal y de Fauna Silvestre de Puno (ATFFS Puno) del Servicio Nacional Forestal y de Fauna Silvestre (SERFOR) en el río Coata. La rana del Titicaca es una especie categorizada como En Peligro Crítico (CR) a nivel nacional. En Peligro a nivel (EN) a nivel internacional, además de estar incluida en el Apéndice I de la Convención CITES desde el 2017. A pesar de la magnitud de este evento de mortalidad, que dio la vuelta mundo, tampoco existió un diagnóstico definitivo de su origen (SERFOR, 2016). Finalmente, en octubre del 2019, el Servicio Nacional Forestal y de Fauna Silvestre (SERFOR) constató la muerte de 119 ejemplares de zambullidor del Titicaca (Rollandia microptera), especie categorizada En Peligro (EN) a nivel nacional e internacional, en el sector Suchipujo de la provincia de Yunguyo - Puno; evento en el que tampoco pudo determinarse la causa de estas muertes (SERFOR, 2019).

\section{GESTIÓN SANITARIA DE LA FAUNA SILVESTRE}

La gestión de la fauna silvestre comprende distintos aspectos orientados a su conservación y uso sostenible en armonía con los ecosistemas de los que provienen. Las amenazas que suponen los patógenos para la conservación de las poblaciones naturales y, por ende, del hombre, resaltan la urgencia de que la gestión sanitaria deba implementarse en un sentido más amplio e integral que el que se realiza actualmente en nuestro país.

En el Perú, la autoridad nacional responsable de la gestión de la fauna silvestre es el SERFOR, organismo técnico especializado adscrito al Ministerio de Agricultura y Riego (MINAGRI), creado mediante la Ley Forestal y de Fauna Silvestre (Ley N²9763) y que inició sus funciones en el 2014. Aunque el SERFOR, en su condición de autoridad nacional forestal y de fauna silvestre, es el encargado de dictar las normas, las estrategias, los planes, los programas, entre otros procedimientos relacionados con la administración y uso sostenible de la flora y fauna silvestre; no posee todas las competencias para realizar la gestión sanitaria integral de estas especies.

Adicionalmente, con un proceso de descentralización en marcha, el MINAGRI ha transferido varias competencias en materia la fauna silvestre a los gobiernos regionales de Amazonas, San Martín, Loreto, Huánuco, Ucayali, Madre de Dios, Ayacucho, Tumbes y La Libertad, por lo que, en estos departamentos, la gestión de la fauna silvestre se encuentra bajo la competencia de las Autoridades Regionales Forestales y de Fauna Silvestre (ARFFS), cabe indicar que en los departamentos donde aún no se ha culminado el proceso de transferencia de funciones en materia de fauna silvestre a los gobiernos regionales, operan las Administraciones Técnicas Forestales y de Fauna Silvestre (ATFFS), que son órganos desconcentrados del SERFOR. La gestión de la fauna silvestre en el ámbito regional impactada por la poca estabilidad laboral del capital humano y la falta de suficientes equipos, materiales y recursos para el desarrollo de las funciones asignadas.

Por su parte, el Servicio Nacional de Sanidad Agraria (SENASA), organismo técnico especializado adscrito al MINAGRI, creado en 1992 mediante el Decreto Ley $N^{\circ} 25902$. Se constituye en la autoridad nacional en sanidad agraria en el país, según lo establecido por el Decreto Legislativo $\mathrm{N}^{\circ} 1059$, y a través de su Dirección de Sanidad Animal específicamente la Subdirección de Análisis de Riesgo y Vigilancia Epidemiológica, se encarga de conducir el Sistema de Vigilancia Epidemiológica de la salud animal en el país. Asimismo, si bien sus funciones están orientadas a desarrollar acciones vinculadas a la prevención, control y erradicación de plagas y enfermedades, 
estas se centran en la seguridad sanitaria de la labor agropecuaria nacional y la inocuidad agroalimentaria. Por ello, a pesar de contar con procedimientos internos para la vigilancia de enfermedades en animales silvestres, sus intervenciones mayoritariamente se centran en bovinos, equinos, caprinos, porcinos, camélidos sudamericanos, aves y abejas.

Asimismo, existe el Servicio Nacional de Áreas Naturales Protegidas por el Estado (SERNANP), organismo público técnico especializado adscrito al Ministerio del Ambiente (MINAM), creado mediante el Decreto Legislativo $\mathrm{N}^{\circ} 1013$ del 2008, el cual se constituye en el ente rector del Sistema Nacional de Áreas Naturales Protegidas por el Estado - SINANPE. Actualmente, el SINANPE está conformado por 75 Áreas Naturales Protegidas de administración nacional y establecidas con carácter definitivo y 9 zonas reservadas. Complementariamente, se suman 25 Áreas de Conservación Regional y 141 Áreas de Conservación Privadas. En conjunto, todas las mencionadas representan el $17,31 \%$ de territorio terrestre nacional. Si bien el SERNANP es la autoridad competente de la administración del patrimonio forestal y de fauna silvestre de las áreas naturales protegidas y sus servicios ambientales, según lo establecido por el Decreto Legislativo $\mathrm{N}^{\circ} 1079$, no cuenta con competencias expresas para realizar la gestión sanitaria de las especies de fauna silvestre existentes al interior de las áreas naturales protegidas.

De otro lado, el Ministerio de Salud es el organismo del Poder Ejecutivo que ejerce la rectoría del Sector Salud. Dentro de su estructura organizacional, de acuerdo a su vigente Reglamento de Organización y Funciones aprobado por el Decreto Supremo $\mathrm{N}^{\circ}$ 008-2017-SA, la Dirección de Prevención y Control de Enfermedades Metaxénicas y Zoonosis es la encargada de identificar y coordinar las intervenciones estratégicas de prevención, control y reducción de riesgos y daños de las enfermedades metaxénicas y otras transmisibles por vectores; así como enfermedades de los animales al hombre (zoonosis), entre otras que afecten a la población. A pesar de los esfuerzos realizados, aún se requiere generar mayor conocimiento (investigaciones) y fortalecer la gestión y las estrategias de intervención con las autoridades locales y otros actores clave en la prevención y control de enfermedades zoonóticas.

Sin perjuicio de lo señalado, cabe mencionar que la gestión de la fauna acuática en nuestro país recae en el Ministerio de la Producción (PRODUCE), organismo del Poder Ejecutivo competente en las materias de pesquería y acuicultura. Si bien PRODUCE tiene competencias de manera compartida con los gobiernos regionales y gobiernos locales, en materia de pesquería artesanal, acuicultura de micro y pequeña empresa, acuicultura de recursos limitados, entre otros, no cuenta con facultades expresas para la gestión sanitaria de las especies que se encuentran bajo el ámbito de su competencia.

Asimismo, el Instituto del Mar del Perú (IMARPE) es un organismo técnico especializado del PRODUCE, orientado a la investigación científica, así como al estudio y conocimiento del mar peruano. De acuerdo a su actual Reglamento de Organización y Funciones, aprobado por la Resolución Ministerial $\mathrm{N}^{\circ} 345-2012-\mathrm{PRODUCE}$, se encarga promover investigaciones científicas de los recursos del mar y de las aguas continentales; en este contexto, interviene en eventos de varamiento y mortandad de fauna marina, básicamente en el trabajo de campo (toma de muestras y análisis de resultados).

Por lo expuesto, urge la necesidad de promover reformas para reorganizar y fortalecer la institucionalidad vinculada a la gestión sanitaria de la fauna silvestre en el Perú, a fin que esta cuente con un enfoque holístico y una acción oportuna estatal frente a cualquier infección o enfermedad emergente o evento similar. En este marco, se deberán contemplar protocolos de actuación y coordinación efectivos en los tres niveles de gobierno, con la participación de actores clave, y la necesaria previsión de condiciones habilitantes y recursos a las instituciones involucradas para el desarrollo óptimo de sus funciones.

Asimismo, estas reformas deberán incluir el fortalecimiento del conocimiento del ciclo biológico de los agentes infectocontagiosos, la relación entre portadores y diseminadores de los agentes zoonóticos, el origen y la diseminación de enfermedades emergentes con repercusión en la salud pública y en la sanidad animal, entre otros.

Sin ir muy lejos, en países de la región como Chile, tiene dentro de su autoridad responsable de sanidad animal, una oficina encargada de regular y fiscalizar todo lo referente a actividades o eventos relacionados a la fauna silvestre de ese país. El Servicio Agrícola y Ganadero (SAG) es el organismo oficial encargado de apoyar el desarrollo de la agricultura, los bosques y la ganadería, a través de la protección y mejoramiento de la salud de los animales; y su Subdepartamento de 
Vida Silvestre del SAG es el responsable de regular y fiscalizar la caza, captura, tenencia, comercio, transporte y bienestar de fauna silvestre, además de la regulación y fiscalización en el control de lagomorfos y roedores.

\section{VIGILANCIA SANITARIA}

Como se señaló, el SENASA posee un Sistema de Vigilancia Epidemiológica cuyo propósito es obtener información actualizada de la problemática nacional referida a incidencia, localización, repercusión y la cuantificación de las enfermedades animales de mayor impacto, para implementar acciones de control y prevención oportuna. El referido sistema tiene dos componentes primordiales: la vigilancia pasiva vinculada con la recopilación permanente de información epidemiológica por actores secundarios como médicos veterinarios de práctica privada, empresas agropecuarias, laboratorios, universidades, Ministerio de Salud, productores pecuarios, entre otros; así como la vigilancia activa, relacionada con la generación de información por medio de toma de muestras y registro de información epidemiológica para obtener un panorama real del estatus zoosanitario de determinadas enfermedades que se constituyen como de riesgo para el comercio internacional de mercancías pecuarias.

Estos esfuerzos se enfocan primordialmente en la producción agropecuaria y de animales de compañía, pero no incluyen la evaluación de eventos como las mortalidades registradas en poblaciones silvestres señaladas en los antecedentes, las implicancias sanitarias en la gestión de la caza (en todas sus modalidades: deportiva, comercial y de subsistencia) o la de los decomisos de especímenes de fauna silvestre mantenidos en cautiverio o comercializados ilegalmente o los centros de cría de fauna silvestre.

Debido a eso, en junio del 2012, la Subdirección de Análisis de Riesgo y Vigilancia Epidemiólogica de la Dirección de Sanidad Animal del SENASA, con apoyo de Wildlife Conservation Society y Animal Plant Health Inspection Service - APHIS de los EEUU, organizaron el seminario-taller "Fortalecimiento del sistema de vigilancia en animales silvestres en el Perú, enfoque Una Salud", con el objetivo de mejorar la calidad de la vigilancia para la detección temprana y control de las principales enfermedades de notificación obligatoria en los animales de fauna silvestre en las Áreas Naturales Protegidas por el Estado y centros privados y en todo lugar donde se crie, maneje, rescate o se comercialice animales silvestres. En esa oportunidad, profesionales de diferentes especialidades (médicos veterinarios, biólogos, ingenieros forestales, etc.), que estaban trabajando con fauna silvestre en sus diferentes modalidades (cautiverio y vida libre), fueron convocados para dar el inicio a una red nacional de vigilancia sanitaria pasiva de la fauna silvestre del Perú, lamentablemente no hubo continuidad y la iniciativa nunca pudo concretarse.

\section{CONCLUSIONES}

Las enfermedades vinculadas con la fauna silvestre tienen importancia para la salud animal y para la salud pública. Cuanto antes se detecte y gestione una enfermedad, mayores serán las oportunidades para su control.

La gestión sanitaria de la fauna silvestre en el Perú debe implementarse en un sentido más amplio e integral que el que se realiza actualmente en nuestro país.

Teniendo en cuenta la situación global actual, urge en nuestro país la necesidad de promover las reformas necesarias para reorganizar y fortalecer la institucionalidad vinculada a la gestión sanitaria de la fauna silvestre, que incluyan protocolos de actuación y coordinación efectivos en los tres niveles de gobierno y la participación de actores clave, además de las condiciones habilitantes y recursos necesarios a las instituciones involucradas para el desarrollo óptimo de sus funciones.

\section{Correspondencia:}

\section{Roberto Elías \\ Correo electrónico: roberto.elias@upch.pe}

\section{REFERENCIAS BIBLIOGRAFICAS}

1. Alves, D. M., Terribile, L. C., \& Brito, D. (2014). The potential impact of white-nose syndrome on the conservation status of North American bats. PloS one, $9(9)$.

2. Babelhadj, B., Di Bari, M. A., Pirisinu, L., Chiappini, B., Gaouar, S., Riccardi, G... Vaccari, G. (2018). Prion Disease in Dromedary Camels, Algeria. Emerging infectious diseases, 24(6), 10291036. DOI: https://doi.org/10.3201/eid2406.172007

3. Barria, M. A., Libori, A., Mitchell, G., \& Head, M. W. (2018). Susceptibility of Human Prion Protein to Conversion by Chronic Wasting Disease Prions. Emerging infectious diseases, 24(8), 1482- 
1489. DOI: https://doi.org/10.3201/eid2408.161888

4. Berenguel RA., Elias RK., Weaver TJ., \& Reading RP. (2016) Chytrid fungus, Batrachochytrium dendrobatidis, in Wild Populations of the Lake Titicaca Frog, Telmatobius culeus, in Peru. Journal of Wildlife Diseases, 52(4), 973-975.

5. Berger L, Roberts AA, Voyles J, Longcore JE, Murray KA, Skerratt L. (2016). History and recent progress on chytridiomycosis in amphibians. Fungal Ecol, 19, 89-99. DOI: 10.1016/j.funeco.2015.09.007

6. Casapía, M., Mamani, E., García, M., Miraval, M., Valencia, P., Quino, A.,...Donaires, L. (2012). Síndrome pulmonar por Hantavirus (Virus Río Mamoré) en la Amazonía Peruana. Revista Peruana de Medicina Experimental y Salud Publica, 29(3), 390-395. Recuperado de: http://www.scielo. org.pe/scielo.php?script $=$ sci_arttext\&pid $=$ S172646342012000300016\&lng=es\&tlng=e

7. Cunningham, A. A., Daszak, P., \& Wood, J. L. (2017). One Health, emerging infectious diseases and wildlife: two decades of progress?. Philosophical Transactions of the Royal Society B: Biological Sciences, 372(1725), 2016-2067.

8. Fisher, M. C., Henk, D. A., Briggs, C. J., Brownstein, J. S., Madoff, L. C., McCraw, S. L., \& Gurr, S. J. (2012). Emerging fungal threats to animal, plant and ecosystem health. Nature, 484(7393), 186-194.

9. Ghersi, B. M., Blazes, D. L., Icochea, E., Gonzalez, R. I., Kochel, T., Tinoco, Y.,...Montgomery, J. M. (2009). Avian influenza in wild birds, central coast of Peru. Emerging infectious diseases, 15(6), 935938. DOI: https://doi.org/10.3201/eid1506.080981

10. Jones, K. E., Patel, N. G., Levy, M. A., Storeygard, A., Balk, D., Gittleman, J. L., \& Daszak, P. (2008). Global trends in emerging infectious diseases. Nature, 451(7181), 990-993.

11. Lam, T. T., Shum, M. H., Zhu, H. C., Tong, Y. G., Ni, X. B., Liao, Y... Guan, Y. (2020). Identifying SARS-CoV-2 related coronaviruses in Malayan pangolins. Nature,583, 282-285. DOI: https://doi. org/10.1038/s41586-020-2169-0

12. Instituto de Mar del Perú - IMARPE. (2012). Mortandad de delfines en el litoral de la costa norte, febrero a abril de 2012. Informe final. Callao: Instituto de Mar del Perú. Recuperado de: http:// www.imarpe.pe/imarpe/archivos/informes/imarpe vfamar_informefinaldelfines_a_1.pdf

13. Lorch, J. M., Knowles, S., Lankton, J. S., Michell, K., Edwards, J. L., Kapfer, J. M.,...\& Blodgett, D. (2016). Snake fungal disease: an emerging threat to wild snakes. Philosophical Transactions of the Royal Society B: Biological Sciences, 371(1709), 20150457.

14. Navarro, A., Bustamante, J. \& Sato, A. (2007). Situación actual y control de la rabia en el Perú. Revista Peruana de Medicina Experimental y Salud Publica, 24(1), 46-50. Recuperado de: http://www.scielo.org.pe/scielo.php?script=sci arttext\&pid $=$ S1726-46342007000100008\&lng=es\&t $\operatorname{lng}=$ es.

15. Organización Mundial de Sanidad Animal. (2009). Cost of National Prevention Systems for Animal Diseases and Zoonoses in Developing and Transition Countries. Paris: Organización Mundial de Sanidad Animal. Recuperado de: https:/www.oie.int/ fileadmin/Home/eng/Conferences_Events/sites/ OIE-WB_Conference_1007/OIE-Costs of National Prevention Systems-final report.pdf

16. Organización Mundial de Sanidad Animal. (2013). Newcastle Disease: Aetiology Epidemiology Diagnosis Prevention and Control References. Paris: Organización Mundial de Sanidad Animal Recuperado de: https://www.oie.int/es/sanidad-animal-en-elmundo/enfermedades-de-los-animales/enfermedadde-newcastle/

17. Organización Mundial de Sanidad Animal. (2018). Manual Terrestre. Chapter 3. 4. 6. Bovine Tuberculosis. Paris: Organización Mundial de Sanidad Animal. Recuperado de: https:/www.oie.int/ fileadmin/Home/eng/Health_standards/ tahm/3.04.06_BOVINE_TB.pdf

18. Organización Mundial de Sanidad Animal. (2019). African Swine Fever: Aetiology Epidemiology Diagnosis Prevention and Control References. Paris: Organización Mundial de Sanidad Animal. Recuperado de: https:/www.oie.int/fileadmin/Home/ eng/Animal_Health_in_the_World/docs/pdf/ Disease_cards/AFRICAN_SWINE_FEVER.pdf

19. Olson, D.H., Aanensen, D.M., Ronnenberg, K.L., Powell, C.I., Walker, S.F., Bielby, J. (2013). Mapping the Global Emergence of Batrachochytrium dendrobatidis, the Amphibian Chytrid Fungus. PLoS ONE, 8(2), e56802. DOI: https://doi. org/10.1371/journal.pone.0056802

20. Race, B., Meade-White, K. D., Phillips, K., Striebel, J., Race, R., \& Chesebro, B. (2014). Chronic wasting disease agents in nonhuman primates. Emerging infectious diseases, 20(5), 833837. DOI: https://doi.org/10.3201/eid2005.130778

21. Rezza G. (2004). Avian influenza: a human pandemic threat? Journal of Epidemiology \& Community Health, 2004, 58:807-808.

22. Segovia H., K., Icochea D., E., González V., R., Ghersi, B., \& González Z., A. (2013). Presencia del virus de influenza aviar en aves silvestres de los humedales de Puerto Viejo, Lima. Revista De Investigaciones Veterinarias Del Perú, 24(1), 98-103. DOI: https://doi.org/10.15381/rivep.v24i1.1672

23. Servicio Nacional Forestal y de Fauna Silvestre. (2016). SERFOR informa la muerte de más de 10 mil ranas en río Coata, Puno. Lima: Servicio Nacional Forestal y de Fauna Silvestre. Recuperado de: $\quad$ https://www.serfor.gob.pe/noticias/fauna- 
silvestre/serfor-evalua-muerte-de-mas-de-10-milranas-en-rio-coata-en-puno

24. Servicio Nacional Forestal y de Fauna Silvestre. (2019). Puno: SERFOR constata muerte de aves zambullidor del Titicaca en la provincia fronteriza de Yunguyo. Lima: Servicio Nacional Forestal y de Fauna Silvestre .Recuperado de: https://www.serfor. gob.pe/noticias/negocios-sostenibles/puno-serforconstata-muerte-de-aves-zambulllidor-del-titicacaen-provincia-fronteriza-de-yunguyo

25. Shi, J., Wen, Z., Zhong, G., Yang, H., Wang, C., Huang, B... Zhao, Y. (2020). Susceptibility of ferrets, cats, dogs, and other domesticated animals to SARScoronavirus 2. Science,368(6494),1016-1020. doi: 10.1126/science.abb701

26. Skerratt, L. F., Berger, L., Speare, R., Cashins, S., McDonald, K. R., Phillott, A. D.,... Kenyon, N. (2007). Spread of chytridiomycosis has caused the rapid global decline and extinction of frogs. EcoHealth, 4(2), 125.

27. Sociedad Peruana de Derecho Ambiental. (2020). SPDA Actualidad Ambiental. Lima: Sociedad Peruana de Derecho Ambiental. Recuperado de: https://www.actualidadambiental.pe/cajamarcaatacan-con-fuego-a-murcielagos-porque-pensaronque-transmitia-coronavirus/

28. Uhart, M., Pérez, A. A., Rostal, M., Robles, E. A., Mendoza, A. P., Nava, A., ... \& Durigon, E. (2013). A 'One Health'Approach to Predict Emerging
Zoonoses in the Amazon. Wildlife and Human Health: Experiences and Perspectives, 65-73.

29. International Union for Conservation of NatureUICN (2009). Wildlife crisis worse than economic crisis. Disponible en: https://www.iucn.org/content/wildlifecrisis-worse-economic-crisis-iucn

30. Viana, M., Cleaveland, S., Matthiopoulos, J., Halliday, J., Packer, C., Craft, M. E., ... \& Ernest, E. (2015). Dynamics of a morbillivirus at the domesticwildlife interface: Canine distemper virus in domestic dogs and lions. Proceedings of the National Academy of Sciences, 112(5), 1464-1469.

31. Weaver, G. V., Domenech, J., Thiermann, A. R., \& Karesh, W. B. (2013). Foot and mouth disease: a look from the wild side. Journal of Wildlife Diseases, 49(4), 759-785.

32. Ye, Z. W., Yuan, S., Yuen, K. S., Fung, S. Y., Chan, C. P., \& Jin, D. Y. (2020). Zoonotic origins of human coronaviruses. International journal of biological sciences, 16(10), 1686.

33. Zevallos S., Elías R.K., Berenguel R.A., Weaver T.J., \& Reading R.P. (2016). Batrachochytrium dendrobatidis in Confiscated Telmatobius in Lima, Peru. Journal of Wildlife Diseases, 52(4), 949-952

34.Zhang, T., Wu, Q., \& Zhang, Z. (2020). Probable pangolin origin of SARS-CoV-2 associated with the COVID-19 outbreak. Curr Biol, 30(7),1346-1351. doi: 10.1016/j.cub.2020.03.022 